\title{
Synergistic anti-tumor activity of acadesine (AICAR) in combination with the anti-CD20 monoclonal antibody rituximab in in vivo and in vitro models of mantle cell lymphoma
}

\author{
Arnau Montraveta ${ }^{1}$, Sílvia Xargay-Torrent ${ }^{1}$, Mónica López-Guerra $^{1}$, Laia Rosich ${ }^{1}$, \\ Patricia Pérez-Galán ${ }^{1}$, Itziar Salaverria ${ }^{1}$, Silvia Beà ${ }^{1}$, Susana G. Kalko ${ }^{2}$, Mercè de \\ Frias $^{3}$, Clara Campàs ${ }^{3}$, Gaël Roué ${ }^{1}$, Dolors Colomer ${ }^{1,4}$ \\ ${ }^{1}$ Experimental Therapeutics in Lymphoid Malignancies Group, Institut d'Investigacions Biomèdiques August Pi i Sunyer \\ (IDIBAPS), Barcelona, Spain, \\ 2 Bioinformatics Core Facility, IDIBAPS, Barcelona, Spain \\ ${ }^{3}$ Advancell-Advanced In Vitro Cell Technologies S.A., Barcelona, Spain \\ ${ }^{4}$ Hematopathology Unit, Hospital Clínic, Barcelona, Spain \\ Correspondence to: Dolors Colomer, email: dcolomer@clinic.cat
}

Gaël Rové, email: grove@clinic.cat

Keywords: Acadesine, rituximab mantle cell lymphoma, xenograft mouse model

Received: September 30, 2013 Accepted: January 23, 2014

Published: January 25, 2014

This is an open-access article distributed under the terms of the Creative Commons Attribution License, which permits unrestricted use, distribution, and reproduction in any medium, provided the original author and source are credited.

ABSTRACT:

Mantle cell lymphoma (MCL) is considered one of the most challenging lymphoma, with limited responses to current therapies. Acadesine, a nucleoside analogue has shown antitumoral effects in different preclinical cancer models as well as in a recent phase I/II clinical trial conducted in patients with chronic lymphocytic leukemia. Here we observed that acadesine exerted a selective antitumoral activity in the majority of MCL cell lines and primary MCL samples, independently of adverse cytogenetic factors. Moreover, acadesine was highly synergistic, both in vitro and in vivo, with the anti-CD20 monoclonal antibody rituximab, commonly used in combination therapy for MCL. Gene expression profiling analysis in harvested tumors suggested that acadesine modulates immune response, actin cytoskeleton organization and metal binding, pointing out a substantial impact on metabolic processes by the nucleoside analog. Rituximab also induced changes on metal binding and immune responses.The combination of both drugs enhanced the gene signature corresponding to each single agent, showing an enrichment of genes involved in inflammation, metabolic stress, apoptosis and proliferation. These effects could be important as aberrant apoptotic and proinflammatory pathways play a significant role in the pathogenesis of MCL. In summary, our results suggest that acadesine exerts a cytotoxic effect in MCL in combination with rituximab, by decreasing the proliferative and survival signatures of the disease, thus supporting the clinical examination of this strategy in MCL patients.

\section{INTRODUCTION}

Mantle cell lymphoma (MCL) represents $5-10 \%$ of all non-Hodgkin lymphomas (NHLs) and is one of the most aggressive lymphoid neoplasms with poor prognosis. Its genetic hallmark is the chromosomal translocation $\mathrm{t}(11 ; 14)(\mathrm{q} 13 ; \mathrm{q} 32)$, which leads to cyclin D1 overexpression with the consequent cell cycle deregulation
[1]. MCL cells carry a high number of secondary genetic alterations that increase the oncogenic potential of cyclin D1 and frequently inactivate the cellular response to DNA damage. In addition, other mechanisms such as activation of cell survival pathways are integrated to drive MCL pathogenesis. Current frontline combination chemotherapies and intensive chemoimmunotherapy followed by stem-cell transplantation have improved the 
outcome for patients with this disease [2]. Although these regimens have high initial response rates, most patients relapse and die from disease-related complications [1]. The introduction of rituximab, a chimeric mouse anti-human CD20 monoclonal antibody, has shown improvement of response rates when used in combination with standard chemotherapy [3;4]. In the last years, new strategies that target crucial biological pathways such as ubiquitinproteasome system, mTOR pathway and BCR signaling have been developed $[2 ; 5 ; 6]$. In particular, recently it has been described that Ibrutinib, a BTK inhibitor shows durable single-agent efficacy in relapsed or refractory MCL [7]. Acadesine (5-aminoimidazole-4-carboxamide-1-Dribofuranoside, AICA-riboside or AICAR) is a nucleoside analogue initially developed as a cardioprotective agent, with a different mechanism of action compared to standard nucleoside analogues, like fludarabine [8]. When added to cell cultures or administered to animals or humans, acadesine is phosphorylated to AICA-ribotide (ZMP), the natural endogenous intermediate in the de novo purine nucleotide biosynthesis, which can function as an AMP mimic and activate AMP-activated kinase (AMPK), a protein that regulates the responses of the cell to energy changes [9]. Although acadesine is commonly used as an AMPK activator, there are compelling evidences that acadesine anti tumoral effects could be mediated, at least in part, independently of the AMPK pathway [10-13]. Nevertheless, at present, the exact nature of the AMPKindependent effects of acadesine in leukemic cells is not clearly understood. Many studies have shown that acadesine can inhibit proliferation, and induce apoptosis in multiple myeloma [14], neuroblastoma [15], glioblastoma [16], childhood acute lymphoblastic leukemia (ALL) [17], colon cancer [18], and breast and prostate cancer cell lines [19]. In particular, acadesine exerts a pro-apoptotic activity in a wide range of B lymphoid malignancies [20], being cells from chronic lymphocytic leukemia (CLL) the most sensitive to this agent $[13 ; 21]$. Recently, a phase I/II clinical trial conducted in relapsed/refractory CLL patients has demonstrated a remarkable activity of the drug in the clinical settings [22].

In this study, we show that acadesine exerts a specific antitumoral activity in the majority of MCL cell lines and primary samples, and synergizes with rituximab both in vitro and in vivo. Furthermore, we provide gene expression profiling (GEP) data on the putative mechanisms of action of acadesine-rituximab combination, supporting clinical examination of this strategy in MCL patients.

\section{RESULTS}

\section{Acadesine exerts a cytotoxic effect both in MCL cell lines and MCL primary samples}

Nine MCL cell lines were exposed for 24 or 48 hours to increasing doses of acadesine ranging from 0.1 to $2 \mathrm{mM}$, and $\mathrm{IC}_{50}$ and $\mathrm{LD}_{50}$ values were calculated from data obtained by the MTT and the Annexin V assays, respectively. As shown on Table 1, most of the cell lines analyzed (REC-1, JEKO-1, UPN-1, JVM-2, MAVER-1 and Z-138) showed a $\mathrm{IC}_{50}$ lower than $1 \mathrm{mM}$ after 48 hours of acadesine incubation. Three cell lines (MINO, HBL-2 and GRANTA-519), showed a $\mathrm{IC}_{50}$ higher than 2 $\mathrm{mM}$, the maximum dose tested. The analysis of the $\mathrm{LD}_{50}$ values followed the same pattern, being REC-1, JEKO1 and UPN-1 the most sensitive cell lines to acadesineinduced cell death, with $\mathrm{LD}_{50}$ below $1 \mathrm{mM}$ after 48 hours of incubation (Figure 1A and 1C). Z-138, JVM-2 and MAVER-1 cell lines showed $\mathrm{LD}_{50}$ around $1.5 \mathrm{mM}$ at 48 hours and, accordingly to the MTT assay results, MINO, HBL-2 and GRANTA-519 exhibited low sensitivity to acadesine with a not-reachable $\mathrm{LD}_{50}$. Acadesine-evoked cell death increased in a time- and a dose-dependent manner, as illustrated in Figure 1A. Deletion at $17 \mathrm{p}$, affecting TP53 or the total amount of copy number alterations (CNA), including trisomies or monosomies that carried most of the MCL cell lines (Table 1) did not affect the susceptibility of MCL cells to acadesine.

Then, isolated tumor cells from $15 \mathrm{MCL}$ samples were exposed for 24 hours to acadesine 1 and $2 \mathrm{mM}$, and cell viability was analyzed by annexin $\mathrm{V}$ labeling. As shown on Table 1 and illustrated on figure 1C, similarly to what observed in MCL cell lines, acadesine also induced apoptosis in primary patient cells, even though this effect was heterogeneous among our set of MCL primary cultures (Figure 1B). Six out of fifteen cases (40\%) showed a response above $25 \%$ to $1 \mathrm{mM}$ acadesine, while 12 of 15 cases $(80 \%)$ achieved these responses at $2 \mathrm{mM}$ acadesine, being the mean cytotoxicity at this dose $48.28 \pm$ $27.97 \%$. Again, no association could be observed between the response to acadesine and the presence of TP53 anomalies and CNAs in the set of primary MCL samples studied. Despite all of them harbored a high percentage of tumoral B-cells (range 76-97\%) (Table 1), we analyzed the activity of acadesine in B-tumoral and the accompanying T-cells in 10 out of the $15 \mathrm{MCL}$ cases studied. Using a triple CD19/CD3/Annexin V labeling, we found that B tumor cells (CD19+) were significantly more sensitive to a $2 \mathrm{mM}$ dose of the drug than the normal T-cell subset, both at 24 and 48 hours (Figure 1D, $P<0.01$ at 24 hours and $P<0.001$ at 48 hours).

Altogether, these results suggest that acadesine is active in the majority of MCL cell lines and primary 
samples, where it exerts a selective antitumoral effect, regardless of genetic alterations and adverse prognostic factors.

\section{Acadesine and rituximab exert a synergistic cytotoxic effect}

We further investigated potential interactions of acadesine with drugs currently approved for the treatment of relapsed/refractory MCL, including bortezomib, bendamustine and rituximab. For this aim, a panel of MCL cell lines were incubated for 48 hours with two different doses of acadesine ( 0.5 and $1 \mathrm{mM})$, bortezomib (2.5 and $5 \mathrm{nM})$ and bendamustine $(25$ and $50 \mu \mathrm{M})$. Rituximab experiments were performed after incubation of cells for $24 \mathrm{~h}$ with acadesine, followed by an additional $24 \mathrm{~h}$ incubation with or without two different concentrations of rituximab (20 and $40 \mu \mathrm{g} / \mathrm{mL}$ ), except for JEKO-1 cells where rituximab was used at 1 and $2 \mu \mathrm{g} / \mathrm{ml}$. Inhibition of proliferation was measured using the MTT assay. Then the combination index (CI) using the Chou and Talalay method were evaluated for each drug combination and represented in Figure 2A. An antagonistic effect was observed when acadesine was combined with $5 \mathrm{nM}$ bortezomib. When used in combination with bendamustine $25 \mu \mathrm{M}$, acadesine displayed either additive or synergistic cytotoxic activity, depending on the MCL cell line, and being the cell lines carrying a P53 wild type phenotype those with the higher synergistic effect between these two drugs. Interestingly, a synergistic effect of acadesine plus rituximab was observed in 7 out of the $9 \mathrm{MCL}$ cell lines

A

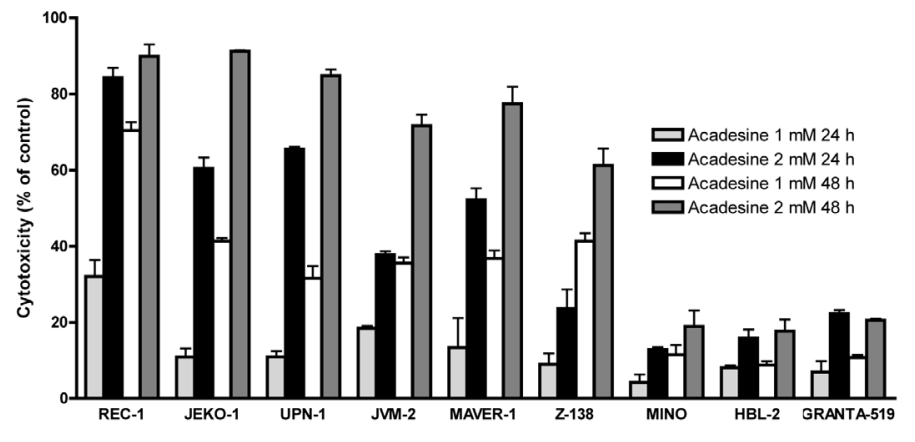

B

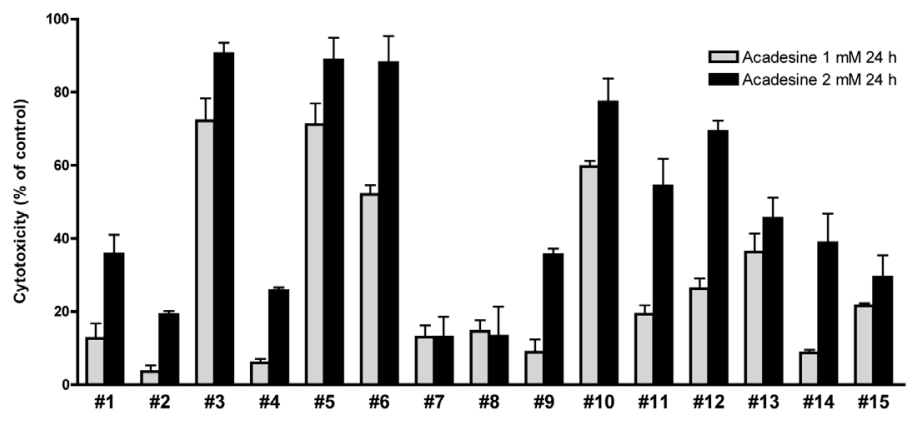

C
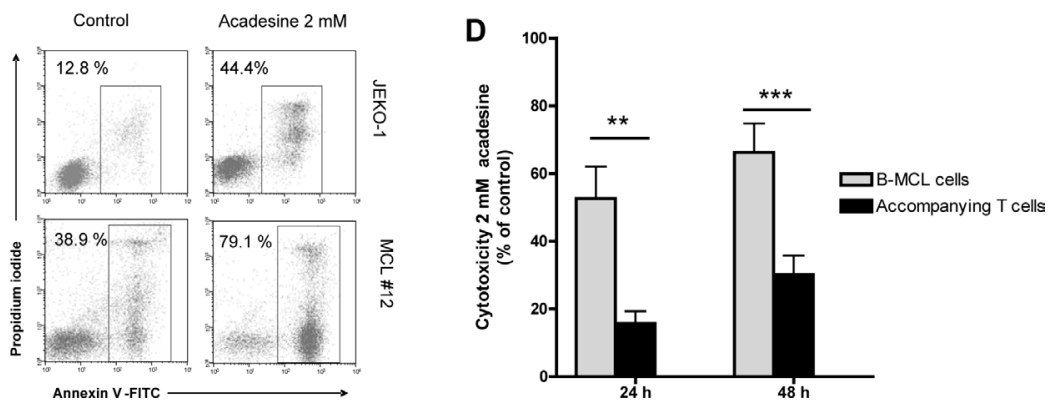

Figure 1: Acadesine induces cytotoxicity in both MCL cell lines and MCL primary samples. A. MCL cell lines were incubated with acadesine $1 \mathrm{mM}$ and $2 \mathrm{mM}$ for 24 and 48 hours and cytotoxicity was measured by Annexin V labeling. Data show the mean \pm SEM of three independent experiments. B. Primary MCL cells were incubated with acadesine $1 \mathrm{mM}$ and $2 \mathrm{mM}$ for 24 hours and cytotoxicity was measured as above. Data show the mean \pm SEM of three replicates. C. Representative flow cytometric plots of Annexin V/ Propidium iodide labeling in a representative MCL cell line (JEKO-1) and a primary MCL sample (MCL\#12) treated with acadesine $2 \mathrm{mM}$ for 24 hours. D. Acadesine cytotoxicity in B tumoral and T normal lymphocytes from MCL cases. Results show the mean cytotoxicity of 10 primary MCL samples \pm SEM analyzed after incubation with acadesine $2 \mathrm{mM}$ for 24 hours. $(* * P<0.01, * * * P<0.001)$ 
TABLE 1. Genetic characteristics of MCL cel lines and MCL primary samples

\begin{tabular}{|c|c|c|c|c|c|c|c|}
\hline \multirow[t]{2}{*}{ MCL cell lines } & \multicolumn{3}{|c|}{ Copy number alterations ${ }^{a}$} & \multirow{2}{*}{$\begin{array}{c}\text { Genetic alterations } \\
\qquad \operatorname{TP53}^{b}\end{array}$} & \multicolumn{2}{|c|}{$\begin{array}{c}\text { Acadesine sensitivity } 48 \\
\text { h }(\mathrm{mM})\end{array}$} & \multirow{2}{*}{$\begin{array}{c}\text { Combination index } \\
\text { Acadesine-Rituximab }^{\mathrm{d}}\end{array}$} \\
\hline & Total & Trisomies & Monosomies & & $\mathrm{IC}_{50}$ & $\mathbf{L D}_{\mathbf{5 0}}$ & \\
\hline REC-1 & 37 & 0 & 0 & wt/mut & 0.28 & 0.65 & 0.822 \\
\hline JEKO-1 & 79 & 4 & 0 & $\mathrm{del} / \mathrm{mut}$ & 0.59 & 1.00 & 0.400 \\
\hline UPN-1 & 47 & 1 & 0 & $\mathrm{del} / \mathrm{mut}$ & 0.64 & 0.78 & 0.786 \\
\hline JVM-2 & 8 & 0 & 0 & wt & 0.98 & 1.51 & 0.918 \\
\hline MAVER-1 & 47 & 0 & 0 & $\mathrm{del} / \mathrm{mut}$ & 0.50 & 1.26 & 1.119 \\
\hline Z-138 & 25 & 3 & 0 & wt & 0.14 & 1.46 & 0.646 \\
\hline MINO & 22 & 3 & 0 & UPD/mut & NR & NR & 0.515 \\
\hline HBL-2 & 65 & 0 & 0 & $\mathrm{del} / \mathrm{mut}$ & NR & NR & 0.813 \\
\hline GRANTA-519 & 30 & 1 & 0 & $\mathrm{del} / \mathrm{wt}$ & NR & NR & 1.154 \\
\hline \multirow{2}{*}{$\begin{array}{l}\text { MCL samples } \\
\text { (\% tumoral cells) }\end{array}$} & \multicolumn{3}{|c|}{ Copy number alterations ${ }^{a}$} & Genetic alterations & \multicolumn{2}{|c|}{$\begin{array}{c}\% \text { Acadesine } \\
\text { cytotoxicity } 24 \mathrm{~h}\end{array}$} & Combination index \\
\hline & Total & Trisomies & Monosomies & $T_{P 53}{ }^{b}$ & $1 \mathrm{mM}$ & $2 \mathrm{mM}$ & Acadesine-Rituximab $^{\mathrm{d}}$ \\
\hline MCL \#1 $(83 \%)$ & 41 & 1 & 0 & $\mathrm{del} / \mathrm{wt}$ & 12.68 & 35.75 & - \\
\hline MCL \#2 (85\%) & 9 & 0 & 1 & wt & 3.58 & 19.22 & - \\
\hline MCL \#3 (97\%) & 0 & 0 & 0 & wt & 72.20 & 90.50 & 0.551 \\
\hline MCL \#4 (89\%) & 2 & 0 & 0 & wt & 6.05 & 25.71 & - \\
\hline MCL \#5 (91\%) & 25 & 0 & 0 & $\mathrm{del} / \mathrm{wt}$ & 71.10 & 88.80 & 0.715 \\
\hline MCL \#6 (86\%) & 13 & 1 & 0 & $\mathrm{del} / \mathrm{mut}$ & 52.04 & 88.71 & - \\
\hline MCL \#7 (86\%) & 14 & 0 & 0 & wt & 12.97 & 13.01 & - \\
\hline MCL \#8 (80\%) & 4 & 0 & 0 & mut & 14.64 & 13.25 & - \\
\hline MCL \#9 (77\%) & 2 & 0 & 0 & $\mathrm{del} / \mathrm{wt}$ & 8.87 & 35.51 & - \\
\hline MCL \#10 (84\%) & 1 & 0 & 0 & $\mathrm{del} / \mathrm{wt}$ & 59.70 & 77.30 & - \\
\hline MCL \#11 (77\%) & 0 & 0 & 0 & wt & 19.31 & 54.30 & - \\
\hline MCL \#12 (76\%) & 15 & 0 & 0 & wt & 26.22 & 69.21 & - \\
\hline MCL \#13 (97\%) & 4 & 0 & 0 & $\mathrm{del} /{ }^{\mathrm{c}}$ & 36.25 & 45.48 & 0.575 \\
\hline MCL \#14 (94\%) & 19 & 0 & 1 & wt & 8.70 & 38.81 & 0.681 \\
\hline MCL \#15 (92\%) & 8 & 0 & 0 & $\mathrm{UPD} /{ }^{c}$ & 21.59 & 29.35 & 0.462 \\
\hline
\end{tabular}

Abbreviations: del, deletion ; mut, mutation; wt, wild type; UPD, uniparental disomy; NR, not reached; ND, not determined

adel and UPD detected by SNP-100K array

${ }^{\mathrm{b}}$ TP53 mutational status detected by direct sequencing

c mutations not analyzed

${ }^{\mathrm{d}}$ Doses used: JEKO-1, acadesine $1 \mathrm{mM}$ and rituximab $1 \mu \mathrm{g} / \mathrm{ml}$; other cell lines and primary samples, acadesine $1 \mathrm{mM}$ and rituximab $40 \mu \mathrm{g} / \mathrm{ml}$

tested, with CI values ranging from 0.400 to 0.918 , with no correlation with any known MCL genetic alteration (Table 1). The two remaining MCL cell lines (MAVER-1 and GRANTA-519), showed CI values closed to 1, indicative of an additive or a slightly antagonistic effect.

In 5 MCL primary samples, the combination of acadesine with rituximab was also synergistic at all the concentrations tested (Table 1), being the best drug interaction obtained with acadesine $1 \mathrm{mM}$ and rituximab $40 \mu \mathrm{g} / \mathrm{ml}$ (mean $\mathrm{CI}=0.597 \pm 0.102$, Figure 2C). Importantly, the synergistic effect observed in primary MCL cells was independent of the initial response to acadesine, being rituximab able to sensitize MCL cells and to overcome their resistance to the nucleoside analog.

To validate the specificity of the cooperation between acadesine and rituximab in MCL, we evaluated the cytotoxic effect of both drugs either alone or in combination in two CLL cell lines, namely MEC-1 and
MEC-2, and in a set of 4 primary CLL cultures. We observed that in CLL cells the acadesine-combination lacked the synergistic activity observed in MCL cells, with CI values ranging from 1.181 to 8.336 , being additive in the MEC-1 cell line and antagonistic in the MEC-2 cell line, as well as in all the CLL primary samples tested (data not shown).

Altogether, these results suggest that among the standard agents currently used in the clinical, rituximab presents the best combinational activity with acadesine, and that this effect may be specific for the MCL model.

\section{The combination of acadesine and rituximab inhibits tumor outgrowth in a mouse xenograft model of MCL}

To validate the synergism between acadesine and rituximab observed in JEKO-1, we evaluated the 

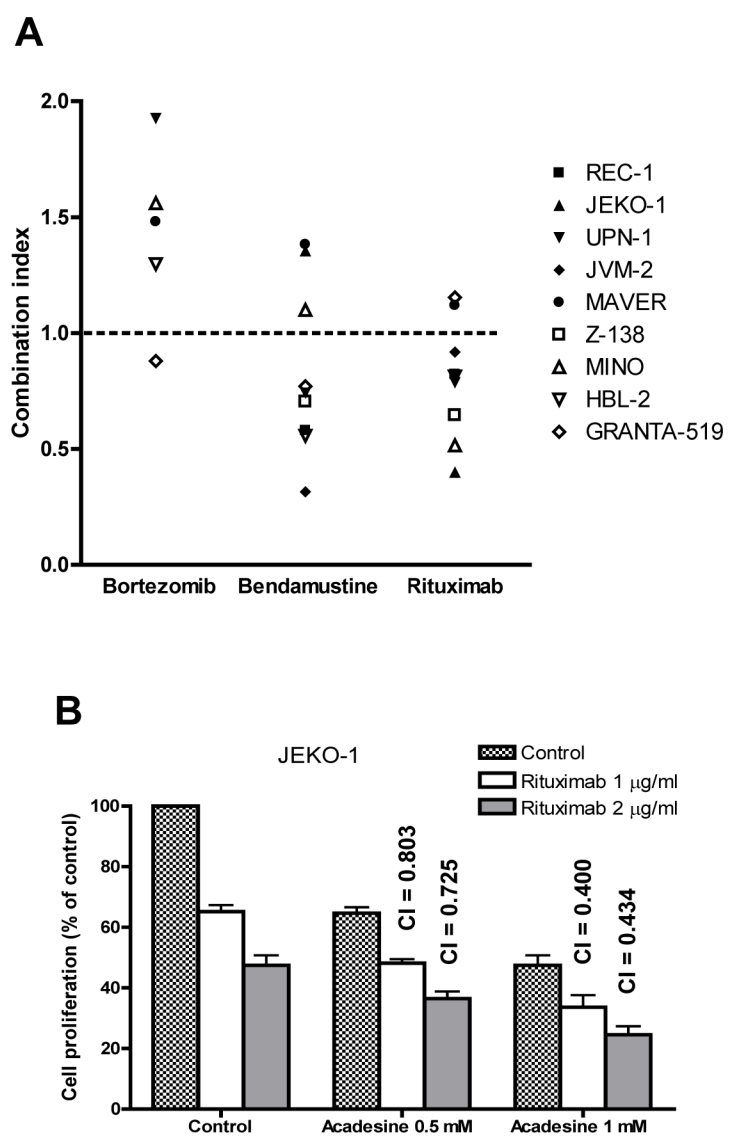

C

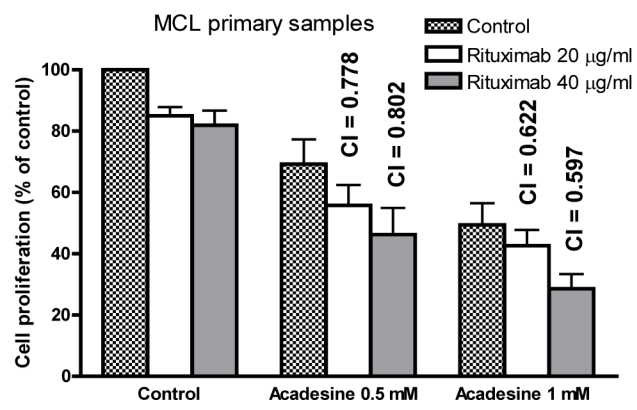

Figure 2: Acadesine shows a synergistic effect with rituximab in vitro and in vivo. A. Acadesine was combined with bortezomib, bendamustine and rituximab in a panel of 9 MCL lines and inhibition of proliferation was analyzed by the MTT assay. The graph shows the CI of the combination of acadesine $1 \mathrm{mM}$ with bortezomib $5 \mathrm{nM}$, bendamustine $25 \mu \mathrm{M}$, and rituximab $40 \mu \mathrm{g} / \mathrm{ml}(1 \mu \mathrm{g} / \mathrm{ml}$ for JEKO-1) for each cell line. The combinations with $\mathrm{CI}>2$ are not shown in the graph. B. Combination of acadesine with rituximab in JEKO-1 cell line. Cells were pre-incubated for 24 hours with acadesine, followed by a 24-hour exposure to rituximab. Inhibition of proliferation was analyzed by the MTT assay. Data show the results of three independent experiments \pm SEM and $\mathrm{CI}$ are indicated above the bars. C. Combination of acadesine with rituximab in MCL primary samples. Primary cells were incubated with acadesine and rituximab as above. Data show the mean of 5 samples \pm SEM and CI are indicated above the bars. antitumoral effect of this combination in a CB17SCID mouse xenograft model of MCL generated using this cell line. Animals were randomly assigned into different groups with 6 mice per cohort and treated with acadesine at dose level of $400 \mathrm{mg} / \mathrm{kg}$ body weight five days a week, rituximab at $10 \mathrm{mg} / \mathrm{kg}$ body weight once a week, both drugs as above or the equivalent volume of vehicle. When compared to vehicle-treated mice, after 18 days of treatment, we observed that tumor burden was significantly reduced in mice treated either with acadesine $(P<0.01)$ or with rituximab $(P<0.001)$ as single agents. We observed a reduction in tumor size of 31.22 $\pm 15.68 \%$ in acadesine-receiving animals and of $63.85 \pm$ $10.88 \%$ in the rituximab arm. The combination of both agents was significantly more effective than the two drugs alone $(P<0.001$ compared to acadesine, $P<0.01$ respect to rituximab) leading to an almost complete inhibition of tumor growth (Figure 3A). Mice tolerated well the treatment, and no effect on animal vital parameters was reported, in any of the treatment arms. Thus, these results suggest that the combination of acadesine with rituximab might represent a safe and efficient therapeutic approach for the treatment of MCL tumors.

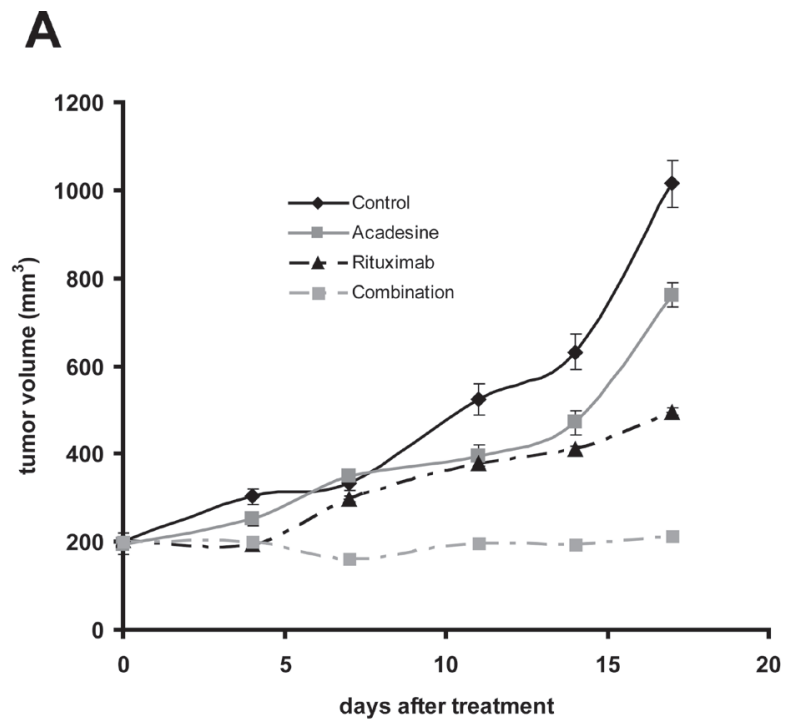

Figure 3: Acadesine-rituximab combination inhibits MCL tumor outgrowth. A. SCID mice were inoculated subcutaneously with JEKO-1 cells and treated with acadesine ( $400 \mathrm{mg} / \mathrm{kg} 5$ days a week), rituximab (10 mg/kg once a week) or both drugs. Tumor growth is represented as the mean \pm SEM $(\mathrm{n}=6)(* * P<0.01, * * * P<0.001)$ 
TABLE 2. GO terms identified with the DAVID functional anotation

\begin{tabular}{|lcccccc|}
\hline \multicolumn{1}{c}{ GO terms } & $\begin{array}{c}\text { Acadesine vs control } \\
\text { Genes }\end{array}$ & P value & $\begin{array}{c}\text { Rituximab vs control } \\
\text { Genes }\end{array}$ & \multicolumn{2}{c}{$\begin{array}{c}\text { Combination vs control } \\
\text { Penes }\end{array}$} & $\begin{array}{l}\text { P value } \\
\text { Metal binding }\end{array}$ \\
\hline Immune response & 8 & $1.7 \times 10-3$ & 20 & $1.3 \times 10-2$ & 202 & $3.3 \times 10-7$ \\
\hline Actin cytoskeleton organization & 4 & $1.4 \times 10-2$ & 7 & $4.8 \times 10-2$ & 78 & $6.9 \times 10-9$ \\
\hline Regulation of cell death & - & NS & - & NS & 64 & $7.2 \times 10-4$ \\
\hline Regulation of cell proliferation & - & NS & - & NS & 63 & $8.6 \times 10-4$
\end{tabular}

DAVID was used to indentify the GO terms related to each treatment. GO terms were considered significant when $P<0.05$

NS, not significant
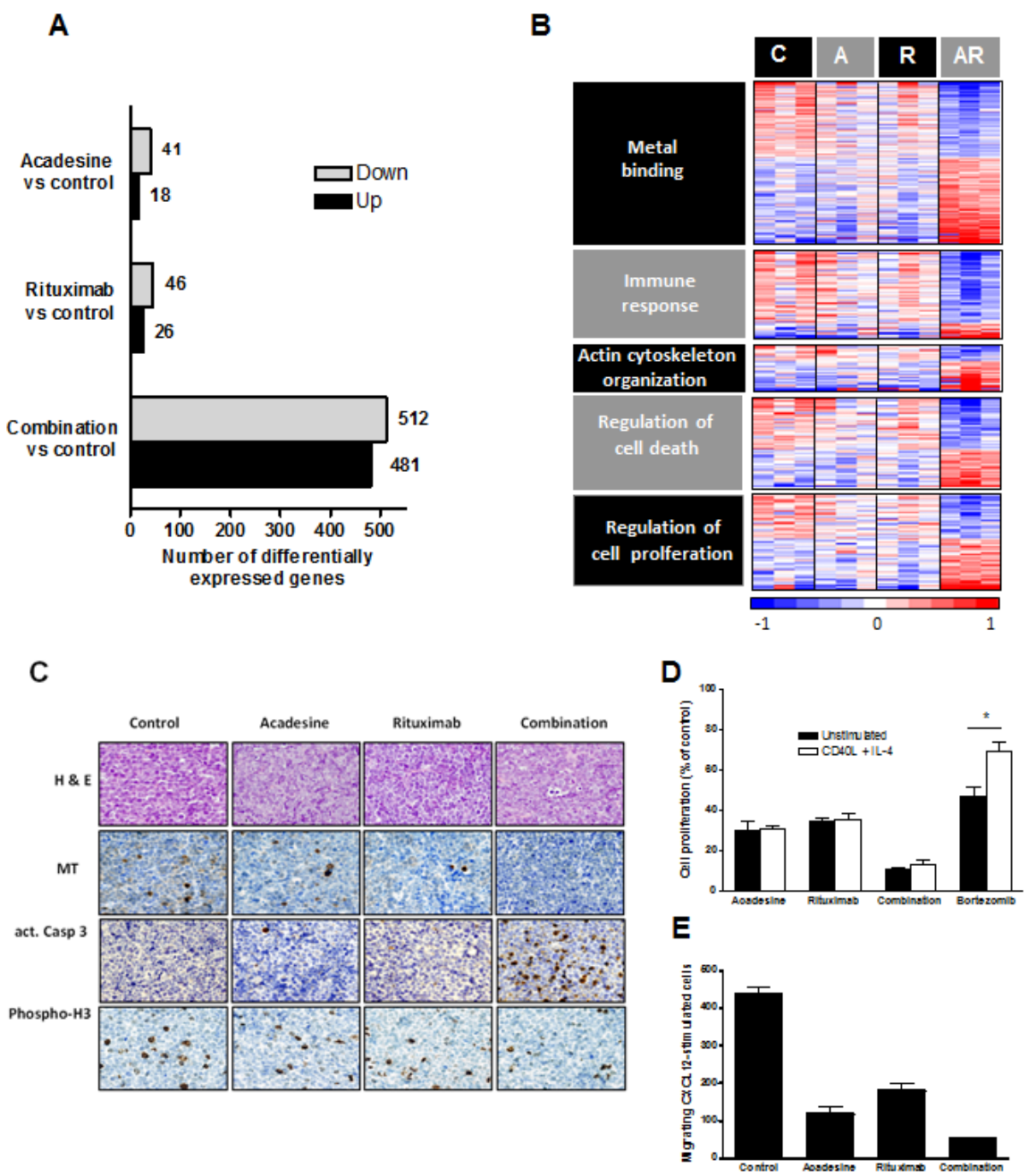

Figure 4: Gene expression profile analysis of mice-treated tumors. GEP analysis of tumors was performed and graph shows the number of differentially expressed genes from each treatment compared to the control with a FDR below 0.2 and an absolute FC above 1.4 B. Heatmap displaying genes modulated by the combination clustered according to GO terms. Relative gene expression levels are color-coded as indicated at the bottom. C: control; A: acadesine; R: rituximab; AR: combination. C. Immunostaining of harvested tumors of mouse xenograft MCL after acadesine, rituximab and acadesine-rituximab treatment. Histological sections from representative tumors of each treatment stained with hematoxylin and eosin and specific antibodies against metallothionein, activated caspase-3 and phosphohistone $3(\mathrm{x} 40)$ D. JEKO-1 cells were treated with acadesine, rituximab and the combination after a CD40L + IL-4 stimulation and inhibition of proliferation was measured by MTT assay. Data show the mean of three independent experiments \pm SEM. E. Migration assay of JEKO-1 cells treated with acadesine, rituximab and the combination. Graph shows the number of CXCL12-stimulated cells migrating after each treatment. 
TABLE 3. Gene sets regulated after treatment with acadesine, rituximab or the combination

\begin{tabular}{lccccccccc}
\multicolumn{1}{c}{ Gene sets } & \multicolumn{3}{c}{ Acadesine vs control } & \multicolumn{3}{c}{ Rituximab vs control } & \multicolumn{3}{c}{ Combination vs control } \\
& Genes & NES & FDR & Genes & NES & FDR & Genes & NES & FDR \\
Interferon pathway & - & - & NS & - & - & NS & 92 & -2.82 & $<1.0 \times 10^{-4}$ \\
B/T cell calcium signaling & - & - & NS & 41 & -1.87 & $1.2 \times 10^{-2}$ & 54 & -2.38 & $<1.0 \times 10^{-4}$ \\
NFkB pathway & - & - & NS & 70 & -1.91 & $1.2 \times 10^{-2}$ & 66 & -2.28 & $<1.0 \times 10^{-4}$ \\
Inflammatory response & - & - & NS & - & - & NS & 23 & -2.02 & $1.4 \times 10^{-3}$ \\
CD40 pathway & - & - & NS & 62 & -1.89 & $1.2 \times 10^{-2}$ & 49 & -1.96 & $2.6 \times 10^{-3}$ \\
Metallothionein node & 4 & -1.92 & $2.8 \times 10^{-2}$ & 5 & -1.86 & $1.2 \times 10^{-2}$ & 5 & -1.96 & $3.5 \times 10^{-3}$ \\
Antiapoptosis & - & - & NS & - & - & NS & 8 & -1.88 & $3.7 \times 10^{-3}$ \\
Metabolic stress & - & - & NS & - & - & NS & 5 & -1.87 & $4.3 \times 10^{-3}$ \\
Toll pathway & - & - & NS & - & - & NS & 9 & -1.83 & $6.0 \times 10^{-3}$ \\
Wnt pathway & - & -1.97 & $2.9 \times 10^{-3}$ & - & - & NS & - & - & NS
\end{tabular}

Gene sets were considered significant when FDR $<0.05$

NES, Normalized Enriched Score; FDR, False discovery rate; NS, not significant

\section{The combination of acadesine and rituximab modulates genes related to inflammation, metabolic stress, proliferation and survival pathways in MCL}

In order to understand the basis of the synergy between acadesine and rituximab, we next examined changes in gene expression profiles in a set of representative harvested tumors from each cohort $(\mathrm{n}=3$ tumors/group). We determined the differentially expressed genes from each treatment compared to the control with a false discovery rate (FDR) below 0.2 and an absolute fold change (FC) above 1.4 using the Rank Products method in the TM4-MEV platform. Treating the cells with acadesine was associated with the lowest number of gene modulations (18 genes up-regulated and 41 genes down-regulated), whereas after rituximab treatment 26 genes were up-regulated and 46 genes were downregulated. Acadesine and rituximab treatment affected the expression of a much higher number of genes, being 481 genes up-regulated and 512 genes down-regulated (Figure 4A and Supplemental Table 1). To gain insight into the biological function of the treatment-related differential expression profiles, we conducted a gene onthology (GO) enrichment analysis using the DAVID application. Both acadesine and rituximab monotherapies were found to be related to an enrichment of genes involved in metal binding and immune response, while genes related to actin cytoskeleton organization and immune response, were more specifically regulated by acadesine and rituximab, respectively. Importantly, the treatment with acadesinerituximab combination allowed to the identification of the same GO terms, but with improved significance $(P<0.001)$. Additionally, an enrichment of genes related to regulation of cell death and cell proliferation was detected in tumors receiving this combination (Table 2). Figure 4B showed an overview of the genes modulated by the combination clustered according to the previous described
GO terms.

Then, GSEA was used to identify the gene-sets and pathways modulated by each treatment considering a FDR $<0.05$ (Table 3). For acadesine, the statistically significant normalized enrichment scores (NES) were achieved by the metallothionein node (NES=-1.92) and the WNT pathway (NES=-1.97). In tumors treated with rituximab, we found also an enrichment of the metallothionein node (NES=-1.86). Furthermore, pathways involved in $\mathrm{B} / \mathrm{T}$ cell calcium signaling $(\mathrm{NES}=-$ $1.87), \mathrm{CD} 40$ (NES $=-1.89)$ and $\mathrm{NF}-\kappa \mathrm{B}$ pathways $(\mathrm{NES}=-$ 1.91) were also down-regulated. A significant regulation of all these pathways, except for WNT, was also observed in tumors treated with the drug combination. Additionally, we observed down-regulation of genes involved in inflammatory response (NES $=-2.02$ ), apoptosis $(\mathrm{NES}=-$ 1.88), metabolic stress (NES $=-1.87$ ), as well as interferon signaling (NES=-2.82) and Toll pathway (NES=-1.83), both of them involved in inflammation (Table 3).

In an attempt to validate the relevance of some of these profiles at the functional level, we first selected to analyze the variation of the metallothionein node, by immunohistochemical detection of metallothionein proteins in the different treatment groups. For this aim, histologic sections from representative whole tumors were labeled with specific antibodies against metallothionein as indicated in material and methods. As shown on figure 4D (panel MT), and in accordance with our GEP results, down-regulation of MT staining was observed in both acadesine- and rituximab-treated groups, that became almost complete in the combination-treated tumors.

Regarding the immune response profile, as the GSEA analysis identified the cytokine receptor CD40 among the main components of this profile, and as it has been reported that CD40 participates in the survival, cell growth, and drug resistance in MCL [23], we performed a co-stimulation assay of JEKO-1 cells with recombinant CD40L + interleukin-4 (IL4), followed by cell exposure to the different drugs and determination 
of cell viability. As shown on figure $4 \mathrm{D}$, stimulation of MCL cells with CD40L+IL4 did not prevent the decrease in cell proliferation either in acadesine, rituximab-, or the combination, contrasting with what observed when MCL cells were incubated with bortezomib. These results suggest that CD40 pro-survival signaling does not affect significantly the acadesine-rituximab cytotoxic activity in MCL cells.

Next, as the actin cytoskeleton organization node contained genes related to the migration of cells, we performed chemotaxis assays in JEKO-1 cells exposed to acadesine, rituximab and the combination. We observed that both agents were able to inhibit the migration of JEKO-1 cells, and that this effect was higher in cells treated with the combination (Figure 4E).

Finally, and in agreement with our DAVID analysis, showing that the regulation of cell death and proliferation were significantly affected by acadesine-rituximab combination in MCL tumors, by immunohistochemistry we observed a higher expression of the activated form of caspase-3 (figure 4C, panel act.Casp3) and a reduction in the phosphorylation status of the proliferation marker histone H3 (figure 4C, panel Phospho-H3), in the tumor tissues treated with acadesine plus rituximab, when compared to vehicle- and single agent-treated tumors.

In summary, the combination of both acadesine and rituximab enhanced the gene signature corresponding to each single agent, showing an enrichment of genes involved in inflammation, metabolic stress, apoptosis and proliferation. These effects could be important as aberrant apoptotic and proinflammatory pathways play an important role in the pathogenesis of MCL.

\section{DISCUSSION}

Acadesine has demonstrated to efficiently block cell proliferation in several tumor models, in association with decreased fatty acid and protein synthesis [24]. Besides inhibiting cell proliferation, acadesine induces also apoptosis in different tumor cell types $[13 ; 15 ; 21 ; 25-$ 27] and autophagy [28]. Recently, the first phase I/II trial has been conducted in relapsed/refractory CLL patients showing a manageable and safe profile and with the conclusion that acadesine may represent a valuable agent for the treatment of this entity [22].

In the present study we show that acadesine exerts a pro-apoptotic effect in the majority of MCL primary cells and cell lines in a time- and dose-dependent manner, at physiologically achievable doses. The $\mathrm{LD}_{50}$ values are slightly higher than those observed in CLL cells, which showed a $\mathrm{LD}_{50}$ of $380 \pm 60 \mu \mathrm{M}$ [21], but similar to those reported previously in a reduced subset of MCL cases [20]. Interestingly, the maximum acadesine dose tested in our experiments $(2 \mathrm{mM})$, did not induce apoptosis in accompanying T-cells from any of the MCL cases analyzed, thus arguing in favor of the specificity of the drug. The same specificity for tumoral B cells have been described in the CLL model [21], that sharply contrasts with the lack of selectivity of other anti-leukemic drugs, like conventional nucleoside analogs [29]. Our results demonstrate that MCL response to acadesine is independent of TP53 status and that it is not affected by the occurrence of CNAs. The situation seems to differ from other cell types, as it has been recently described that acadesine elicited a selective apoptotic response in trisomic mouse embryonic fibroblasts [30] and chromosomal instability-driven colorectal cancer cell lines [31].

The clinical course of MCL is characterized by an initial high response rate but a constant relapse pattern, resulting in a poor long-term outcome [2]. It has been reported that first-line chemotherapy including rituximab is associated with significantly improved survival in older patients diagnosed with MCL compared with chemotherapy alone [3]. In the last years, the proteasome inhibitor bortezomib and bendamustine, an hybrid drug between a nucleoside analog and an alkylating agent, have been approved in USA for the treatment of patients with relapsed MCL patients, either alone or in combination with rituximab [2]. Here, we observed an antagonistic effect between bortezomib and acadesine whereas for bendamustine we detected an additive or synergistic effect depending on the MCL cell line. Our results demonstrated that rituximab is the best useful complementary drug to use in combination with acadesine, compared with bendamustine and bortezomib. The efficacy of the acadesine-rituximab combination was independent of TP53 mutational status in MCL cells, whereas the synergism effect of acadesine plus bendamustine was higher in cell lines with P53 wild type phenotype, consistently with the known role of P53 in MCL cell response to bendamustine [32]. Importantly, this synergistic effect between acadesine and rituximab appeared to be specific for MCL, as it was not observed in CLL cells. The synergistic in vitro effect between acadesine and rituximab was then confirmed in vivo using a xenograft mouse model, where a remarkable regression of the tumor was observed in animals dosed with the drug combination. The antineoplastic effect of acadesine alone has already been reported on the growth of mice xenograft models of prostatic tumors [19], CML [28] and retinoblastoma [33]. Our in vivo results using a xenograft model showed that the treatment with acadesine had a modest anti tumoral activity, suggesting that this drug alone could have limited therapeutic application as a single agent. In contrast, an important anti tumoral activity was observed when combined with rituximab, leading to almost complete inhibition of tumor growth. These results are in agreement with the notion that rituximab significantly improves the response rates in MCL patients when used in combination with standard chemotherapy [2-4], as well as with the last agents approved for the 
treatment of relapsed/refractory MCL patients, like bortezomib [34], temsirolimus [35], and lenalidomide [36]. Based on our present results, acadesine-rituximab may also warrant potential use in the clinical practice.

To date, there is still no absolute consensus of the in vivo mechanism of action of rituximab or rituximabbased combination. Based on in vitro studies, rituximab appears to mediate the depletion of B-cells by several mechanisms that are dependent on the host immune system, including complement-dependent and antibodydependent cellular cytotoxicities [37-39]. It has also been proposed that rituximab might sensitize lymphoma cells to chemotherapy, and have direct antiproliferative and apoptotic effects [38]. Using a GEP approach, we aimed to know the signaling pathways by which acadesine exerts its anti-tumor effect in combination with rituximab. We observed that the number of genes down- and up-regulated after acadesine and rituximab treatments as single agents was low, but that it increased notably with the combination treatment in line with the observed tumor burden inhibition. We found 5 GO terms differentially expressed: metal binding, immune response, actin cytoskeleton organization and regulation of cell death and proliferation. Acadesine alone was able to modulate immune response, actin cytoskeleton organization and metal binding, having a substantial impact of the drug toward metabolic processes, accordingly to a recent microRNA analysis in acadesine-exposed hepatocytes [40]. Rituximab also induced changes on metal binding and immune responses. Accordingly, it has been reported that rituximab was capable of inducing a calcium flux in B-cells through its ability to associate with B-cell receptor (BCR) [41]. CD20 activity is also known to be associated with BCR activation through the induction of Src family tyrosine kinases and the activation of the MAPK pathway [42]. Conversely, rituximab has been shown to inhibit BCR signaling, and different signaling pathways involving MAPK, PI3K/ Akt, NF- $\kappa \mathrm{B}$ and mTOR kinases [43]. Among the metal binding core of genes, we observed a decrease in genes implicated in response to metal ion and negative regulation of cell growth, namely $M T 1 G$ and $M T 1 X$, that codify for two members of the metallothionein family, whose expression has been reported to constitute an independent risk factor in diffuse large B cell lymphoma [44]. We confirmed by inmunohistochemistry the downregulation of these metallothionein proteins in tumors from the three treatment arms, but especially in tumors having received the combination of drugs. Similarly, when considering the immune response profile in the combo group by GEP analysis, we observed an accentuated downregulation of the CD40 signaling pathway. It has been described that the microenvironment, including cytokines, has a central role on MCL cell survival and drug resistance, and that the CD40 system acts as a growth-promoting stimulus [23]. Thus, pharmacological interference with this signaling pathway may have therapeutical relevance in MCL, as demonstrated by the use of the CD40-targeting antibody dacetuzumab in combination with rituximab in in vitro and in vivo models of MCL [45]. Of special relevance, we observed that acadesine, rituximab and their combination were able to overcome the CD40 pro-survival effect in MCL cells, offering a glimpse for further combination therapy in this model. Finally, our results also showed that acadesine and acadesine-based combination interfere with migration in MCL cells. Accordingly, it has been reported that acadesine significantly inhibits the growth of tumors in nude mice xenotransplants of retinoblastoma, by inducing apoptosis and suppressing tumor angiogenesis and macrophage infiltration [33]. Of importance, acadesine and rituximab combination therapy resulted in enhanced cell death in MCL cell lines and primary tumor samples and increased regression of tumor burden in an in vivo murine model of MCL.

In summary, our GEP analysis, together with our histological and functional validation studies, suggest that by mainly decreasing the proliferative and survival gene signatures that drive MCL cell growth, the combination strategy associating rituximab to acadesine may represent a new effective therapy to improve MCL patients' outcome and to reduce treatment-associated toxicities.

\section{METHODS}

\section{Cell lines}

Nine human MCL cell lines (GRANTA-519, JVM2, JEKO-1, Z-138, MAVER-1, REC-1, UPN-1, HBL-2 and MINO) and two CLL cell lines (MEC-1 and MEC-2) were cultured with RPMI 1640, DMEM or IMDM media complemented with 10-20\% heat-inactivated fetal bovine serum (FBS), $2 \mathrm{mM}$ L-glutamine, $50 \mu \mathrm{g} / \mathrm{ml}$ penicillin/ streptomycin (Life Technologies). Cells were grown in a humidified atmosphere at $37 \mathrm{C}^{\circ}$ with $5 \%$ carbon dioxide and routinely tested for Mycoplasma absence by PCR. Additionally, the genetic identity of all cell lines was verified periodically using the AmpFISTR identifier kit (Life Technologies). Genetic characterization of the MCL cell lines is shown in Table 1.

\section{Primary cultures}

Primary cells were obtained from peripheral blood samples of leukemic MCL and CLL patients diagnosed according the WHO criteria. The study was done in accordance with protocols approved by the Ethic Committee of the Hospital Clinic of Barcelona. All patients signed an informed consent according to the Declaration of Helsinki. Biological characteristics of MCL cases are shown in Table 1. Mononuclear cells were isolated by centrifugation on a Ficoll-Hypaque 
(GE Healthcare) gradient and conserved within the Hematopathology collection of our institution. Cells were either used directly or cryopreserved in liquid nitrogen in the presence of $10 \%$ dimethyl sulfoxide, $60 \%$ FBS and $30 \%$ RPMI 1640. Cells were cultured in a supplemented RPMI medium likewise cell lines.

\section{Assays of cytotoxicity}

MCL cell lines were incubated with acadesine (kindly provided by Advancell) at doses ranging from 0.1 to $2 \mathrm{mM}$ for 24 or 48 hours. Inhibition of proliferation was measured using the MTT assay and the $\mathrm{IC}_{50}$ was defined as the concentration of drug required to reduce cell proliferation by $50 \%$. Cell viability was quantified after dual staining of cells with annexin V-fluorescein isothiocyanate (FITC)-conjugated and propidium iodide (PI) (Bender Medsystems), followed by flow cytometry analysis on a FACScan flow cytometer using CellQuest and Paint-A-Gate softwares (Becton Dickinson). Lethal dose $50\left(\mathrm{LD}_{50}\right)$ was defined as the concentration of drug required to reduce cell viability by $50 \%$.

Primary MCL cells were incubated with acadesine at two different doses (1 and $2 \mathrm{mM}$ ) for 24 hours. In these samples, the viability of tumoral B (CD19+) and accompanying $\mathrm{T}(\mathrm{CD} 3+)$ cells was simultaneously analyzed by triple labeling of the samples with anti-CD19phycoerythrin (PE) and anti-CD3-FITC antibodies (Becton Dickinson), and allophycocyanin (APC)-conjugated annexin V (Bender Medsytems) on a FACScalibur flow cytometer.

For bendamustine and bortezomib combinations, MCL lines were incubated simultaneously with acadesine ( 0.5 and $1 \mathrm{mM})$, and bortezomib (2.5 and $5 \mathrm{nM}$ ) or bendamustine ( 25 and $50 \mu \mathrm{M}$ ) for 48 hours. Cytotoxicity was analyzed by the MTT assay. Combination indexes (CI) were analyzed with Calcusyn software (Biosoft), based in the Chou \& Talalay's method [46]. Drug combinations with a CI lower than 1.0 were considered as synergistic.

For acadesine-rituximab studies, cell lines were pre-incubated for 24 hours with acadesine $(0.5$ and 1 $\mathrm{mM}$ for MCL cells and CLL cell lines, 0.1 and 0.25 for CLL primary cases), followed by a 24 hour exposure to 20 and $40 \mu \mathrm{g} / \mathrm{ml}$ of rituximab ( 1 and $2 \mu \mathrm{g} / \mathrm{ml}$ for JEKO1 cells). Because rituximab induces cytotoxicity in part by a complement-mediated mechanism [39], we added $10 \%$ of human $\mathrm{AB}$ plasma together with rituximab as a source of complement proteins. Cytotoxicity and CI values were quantified as above. When indicated, cells were exposed to $1 \mu \mathrm{g} / \mathrm{ml}$ of recombinant human soluble CD40L (rhsCD40L, Sigma) and $20 \mathrm{ng} / \mathrm{ml}$ IL-4 (R\&D Systems) 1 $\mathrm{h}$ before acadesine exposure.

\section{Xenograft mouse model}

Female 6 to 8-week-old CB17-severe combined inmunodeficiency (SCID) mice (Charles River Laboratories Inc) were bred under pathogen-free conditions at the animal facility of our institution using a protocol approved by the Animal Testing Ethical Committee of the University of Barcelona (CEAA).

Mice were inoculated subcutaneously in the right flank with $1 \times 10^{7}$ JEKO-1 cells in Matrigel ${ }^{\mathrm{TM}}$ Basement Membrane Matrix (1:1) (Becton Dickinson). When tumors were palpable and reached a volume of approximately $200 \mathrm{~mm}^{3}$, animals were randomly assigned into different groups with 6 mice per cohort and treated with acadesine at dose level of $400 \mathrm{mg} / \mathrm{kg}$ body weight five days a week, rituximab at $10 \mathrm{mg} / \mathrm{kg}$ body weight once a week, both drugs as above or the equivalent volume of vehicle, for 18 days. The shortest and longest diameters of the tumor were measured with external calipers twice a week. Tumor volume was calculated using the following standard formula: (the shortest diameter) ${ }^{2} \mathrm{x}$ (the longest diameter) $x$ 0.5. Mice were sacrificed at the end of the treatment according to institutional guidelines. Harvested tumors were snap-frozen in Tissue-Tek ${ }^{\circledR}$ O.C.T. medium (Sakura Tissue Tek) or formalin-fixed before paraffin embedding on silane-coated slides in a fully automated immunostainer (Bond Max; Vision Biosystems).

\section{Gene expression studies}

Total RNA was extracted from 3 representative harvested tumors of each group, using the RNeasy Mini Kit (Qiagen). RNA quality and quantity was determined using a 2100 Bioanalyzer (Agilent Technologies) and only high quality RNA samples were processed to Affymetrix GeneChip HT HG-U219 arrays. Sample preparation for microarray hybridization was performed following Affymetrix's protocols. Briefly, from $150 \mathrm{ng}$ of total RNA, a biotin labeled cRNA was generated by reverse transcription. Following cRNA fragmentation, the sample was hybridized on the GeneChip HT HG-U219 perfect-match-only (PM) Array Plate. After hybridization and washes, scanning was processed in the Gene Titan instrument, a fully automated array system. The analysis of the scanned images and the determination of the signal value for each probe set of the array were obtained with GeneChip ${ }^{\circledR}$ Command Console ${ }^{\circledR}$ Software (AGCC) (Affymetrix). Raw data were normalized using the Robust Multichip Analysis (RMA) algorithm of the BioConductor Affy Package.

Differential expression data analysis was carried out using the Multiexperiment Viewer Platform (TM4$\mathrm{MEV}$ ) [47]. To compare each treatment to the control, we determined the number of statistically significant up- and down-regulated genes using Rank Products methodology 
[48] setting up a false discovery rate (FDR) below 0.2 and an absolute fold change (FC) above 1.4.

Gene function was assigned based on Database for Annotation, Visualization and Integrated Discovery (DAVID) tool (http://www.david.abcc.ncifcrf.gov) and Gene Ontology (http://www.geneontology.org) in terms of biological process and molecular function. Gene sets with a $P$ value below 0.05 were considered as significant. Primary microarray data are available at the Gene Expression Omnibus (GEO) of the National Center for Biotechnology Information (GSE47871).

An enrichment pathway analysis was done using the gene set enrichment analysis (GSEA) desktop application version 2.0 (GSEA, Broad Institute at MIT, Cambridge, MA; http://www.broadinstitute.org/gsea/) in order to find significant gene signatures using experimentally derived custom gene sets. Gene sets were downloaded from http:// lymphochip.nih.gov/signaturedb/index.html. A twoclass analysis with 1,000 permutations of gene sets and a weighted metric was used. Gene sets with FDR below 0.05 were considered as significant.

\section{Immunohistochemistry staining}

Immunohistochemical staining studies were performed as previously described [49]. The following antibodies were used: anti-metallothionein (Dako) that recognizes the metallothionein isoforms 1 and 2, anticleaved caspase-3 (Cell Signaling Technology) and antiphospho-Histone H3 (Epitomics). Preparations were evaluated with an Olympus DP70 microscope by means of a $40 \times / 0.75$ NA objective and DPManager software v2.1.1 (Olympus).

\section{Chemotaxis studies}

JEKO- 1 cells $\left(10^{7}\right.$ cells $\left./ \mathrm{mL}\right)$ were washed twice and serum-starved for 1.5 hours in FBS-free RPMI. Acadesine $1 \mathrm{mM}$ and rituximab $2 \mu \mathrm{g} / \mathrm{ml}$ were added simultaneously for 3 additional hours, and cells were diluted to $5 \times$ $10^{6}$ cells $/ \mathrm{mL}$ with $0.5 \%$ bovine serum albumin (BSA; Sigma $)$ in RPMI. One hundred microliters $\left(5 \times 10^{5}\right.$ cells $)$ was added to the top chamber of a Transwell culture polycarbonate insert with $6.5-\mathrm{mm}$ diameter and $5 \mu \mathrm{m}$ of pore size (Corning). Inserts had been previously transferred to wells containing $600 \mu \mathrm{L}$ of RPMI with 200 $\mathrm{ng} / \mathrm{mL}$ of human recombinant CXCL12 (Peprotech). After 3 hours of incubation at $37^{\circ} \mathrm{C}, 100 \mu \mathrm{L}$ was collected in triplicate from each lower chamber and counted by flow cytometry (Attune, Life technologies) for 12 seconds under constant flow rate. Migration is represented as the number of CXCL12-stimulated migrating cells.

\section{Statistical analysis}

All statistical analysis were performed using GraphPad Prism 4.0 Software (Graphpad Software). Comparison of means between two groups of samples was evaluated by non-parametric Mann-Whitney test. Results were considered statistically significant when $P \leq 0.05$ $(* P<0.05, * * P<0.01, * * * P<0.001)$.

\section{ACKNOWLEDGEMENTS}

This study was supported by grants from Spanish Ministry of Economy and Competitiveness (MINECO) (SAF 09/9503, SAF 12/31242 and IPT2012-0673010000), Redes Temáticas de Investigación Cooperativa de Cáncer from the Instituto de Salud Carlos III (ISCIII), MINECO \& European Regional Development Fund (ERDF) "Una manera de hacer Europa” (RD2006/20/014, RD2006/20/039, RD12/0036/0004 and RD12/0036/0036) and Generalitat de Catalunya 2009SGR967. AM is a recipient of FPI predoctoral fellowship from Ministerio de Ciencia e Innovación. ML-G holds a contract from Fundación Científica de la Asociación Española Contra el Cáncer. IS holds a contract from Juan de la Cierva program. PP-G holds a contract from Ramón y Cajal program (RYC2009-05134) and a grant from Ministerio de Ciencia e Innovación (SAF 11/29326). GR holds a contract from Miguel Servet program and grants from Fondo de Investigación Sanitaria (PI09/00060; PI12/01847). The authors thank Laura Jiménez, Jocabed Roldán and Sandra Cabezas for their technical support. This work was carried out at the Esther Koplowitz Center, Barcelona. Acadesine was kindly provided by Advancell.

\section{AUTHORSHIP AND DISCLOSURES}

A Montraveta contributed to the design and conception of the study, performed the research, analyzed and interpreted the data and contributed to write the manuscript. S Xargay-Torrent, M López-Guerra, L Rosich and P Pérez-Galán performed the research, analyzed and interpreted the data. I Salaverria and S Bea participated in the cytogenetics of cell lines and primary MCL tumors. S Kalko participated in the interpretation of microarray data. $\mathrm{M}$ Frias and $\mathrm{C}$ Campàs provided acadesine and critically reviewed the manuscript. G Roue and D. Colomer contributed to the design and conception of the study, interpretation of data and to write the paper.

$\mathrm{M}$ Frias and C Campàs are employed by AdvancellAdvanced In Vitro Cell Technologies SA. The remaining authors declare no competing financial interests.

\section{REFERENCES}

1. Jares P, Colomer D and Campo E. Molecular pathogenesis 
of mantle cell lymphoma. J Clin Invest. 2012; 122(10):3416-3423.

2. Deng C, Lee S and O'Connor OA. New strategies in the treatment of mantle cell lymphoma. Clin Cancer Res. 2012; 18(13):3499-3508.

3. Griffiths R, Mikhael J, Gleeson M, Danese M and Dreyling M. Addition of rituximab to chemotherapy alone as firstline therapy improves overall survival in elderly patients with mantle cell lymphoma. Blood. 2011; 118(18):48084816.

4. Rummel MJ, Niederle N, Maschmeyer G, Banat GA, von GU, Losem C, Kofahl-Krause D, Heil G, Welslau M, Balser C, Kaiser U, Weidmann E, Durk H, Ballo H, Stauch $\mathrm{M}$, Roller F, et al. Bendamustine plus rituximab versus CHOP plus rituximab as first-line treatment for patients with indolent and mantle-cell lymphomas: an open-label, multicentre, randomised, phase 3 non-inferiority trial. Lancet. 2013; 381(9873):1203-1210.

5. Brett LK and Williams ME. Current and emerging therapies in mantle cell lymphoma. Curr Treat Options Oncol. 2013; 14(2):198-211.

6. Perez-Galan P, Dreyling M and Wiestner A. Mantle cell lymphoma: biology, pathogenesis, and the molecular basis of treatment in the genomic era. Blood. 2011; 117(1):26-38.

7. Wang ML, Rule S, Martin P, Goy A, Auer R, Kahl BS, Jurczak W, Advani RH, Romaguera JE, Williams ME, Barrientos JC, Chmielowska E, Radford J, Stilgenbauer S, Dreyling M, Jedrzejczak WW, et al. Targeting BTK with ibrutinib in relapsed or refractory mantle-cell lymphoma. N Engl J Med. 2013; 369(6):507-516.

8. Van Den NE, Van den BG and Bontemps F. AICA-riboside (acadesine), an activator of AMP-activated protein kinase with potential for application in hematologic malignancies. Expert Opin Investig Drugs. 2010; 19(4):571-578.

9. Drew BG and Kingwell BA. Acadesine, an adenosineregulating agent with the potential for widespread indications. Expert Opin Pharmacother. 2008; 9(12):21372144.

10. Jacobs RL, Lingrell S, Dyck JR and Vance DE. Inhibition of hepatic phosphatidylcholine synthesis by 5-aminoimidazole-4-carboxamide-1-beta-4-ribofuranoside is independent of AMP-activated protein kinase activation. J Biol Chem. 2007; 282(7):4516-4523.

11. Kuo CL, Ho FM, Chang MY, Prakash E and Lin WW. Inhibition of lipopolysaccharide-induced inducible nitric oxide synthase and cyclooxygenase- 2 gene expression by 5-aminoimidazole-4-carboxamide riboside is independent of AMP-activated protein kinase. J Cell Biochem. 2008; 103(3):931-940.

12. Guigas B, Bertrand L, Taleux N, Foretz M, Wiernsperger N, Vertommen D, Andreelli F, Viollet B and Hue L. 5-Aminoimidazole-4-carboxamide-1-beta-D-ribofuranoside and metformin inhibit hepatic glucose phosphorylation by an AMP-activated protein kinase-independent effect on glucokinase translocation. Diabetes. 2006; 55(4):865-874.

13. Santidrian AF, Gonzalez-Girones DM, Iglesias-Serret D, Coll-Mulet L, Cosialls AM, de FM, Campas C, GonzalezBarca E, Alonso E, Labi V, Viollet B, Benito A, Pons $\mathrm{G}$, Villunger A and Gil J. AICAR induces apoptosis independently of AMPK and p53 through up-regulation of the BH3-only proteins BIM and NOXA in chronic lymphocytic leukemia cells. Blood. 2010; 116(16):30233032 .

14. Baumann P, Mandl-Weber S, Emmerich B, Straka C and Schmidmaier R. Activation of adenosine monophosphate activated protein kinase inhibits growth of multiple myeloma cells. Exp Cell Res. 2007; 313(16):3592-3603.

15. Garcia-Gil M, Bertini F, Pesi R, Voccoli V, Tozzi MG and Camici M. 5 '-Amino-4-imidazolecarboxamide riboside induces apoptosis in human neuroblastoma cells via the mitochondrial pathway. Nucleosides Nucleotides Nucleic Acids. 2006; 25(9-11):1265-1270.

16. Guo D, Hildebrandt IJ, Prins RM, Soto H, Mazzotta MM, Dang J, Czernin J, Shyy JY, Watson AD, Phelps M, Radu CG, Cloughesy TF and Mischel PS. The AMPK agonist AICAR inhibits the growth of EGFRvIII-expressing glioblastomas by inhibiting lipogenesis. Proc Natl Acad Sci U S A. 2009; 106(31):12932-12937.

17. Sengupta TK, Leclerc GM, Hsieh-Kinser TT, Leclerc GJ, Singh I and Barredo JC. Cytotoxic effect of 5-aminoimidazole-4-carboxamide-1-beta-4-ribofuranoside (AICAR) on childhood acute lymphoblastic leukemia (ALL) cells: implication for targeted therapy. Mol Cancer. 2007; 6:46.

18. Su RY, Chao Y, Chen TY, Huang DY and Lin WW. 5-Aminoimidazole-4-carboxamide riboside sensitizes $\mathrm{T}$. Mol Cancer Ther. 2007; 6(5):1562-1571.

19. Swinnen JV, Beckers A, Brusselmans K, Organe S, Segers J, Timmermans L, Vanderhoydonc F, Deboel L, Derua R, Waelkens E, De SE, Van de ST, Noel A, Foufelle F and Verhoeven G. Mimicry of a cellular low energy status blocks tumor cell anabolism and suppresses the malignant phenotype. Cancer Res. 2005; 65(6):2441-2448.

20. Campas C, Santidrian AF, Domingo A and Gil J. Acadesine induces apoptosis in B cells from mantle cell lymphoma and splenic marginal zone lymphoma. Leukemia. 2005; 19(2):292-294.

21. Campas C, Lopez JM, Santidrian AF, Barragan M, Bellosillo B, Colomer D and Gil J. Acadesine activates AMPK and induces apoptosis in B-cell chronic lymphocytic leukemia cells but not in T lymphocytes. Blood. 2003; 101(9):3674-3680.

22. Van Den NE, Cazin B, Janssens A, Gonzalez-Barca E, Terol MJ, Levy V, Perez de OJ, Zachee P, Saunders A, de FM and Campas C. Acadesine for patients with relapsed/refractory chronic lymphocytic leukemia (CLL): a multicenter phase I/II study. Cancer Chemother Pharmacol. 2013; 71(3):581591. 
23. Andersen NS, Larsen JK, Christiansen J, Pedersen LB, Christophersen NS, Geisler CH and Jurlander J. Soluble CD40 ligand induces selective proliferation of lymphoma cells in primary mantle cell lymphoma cell cultures. Blood. 2000; 96(6):2219-2225.

24. Rattan R, Giri S, Singh AK and Singh I. 5-Aminoimidazole4-carboxamide-1-beta-D-ribofuranoside inhibits cancer cell proliferation in vitro and in vivo via AMP-activated protein kinase. J Biol Chem. 2005; 280(47):39582-39593.

25. Persons KS, Eddy VJ, Chadid S, Deoliveira R, Saha AK and Ray R. Anti-growth effect of 1,25-dihydroxyvitamin D3-3-bromoacetate alone or in combination with 5-aminoimidazole-4-carboxamide-1-beta-4-ribofuranoside in pancreatic cancer cells. Anticancer Res .2010; 30(6):18751880.

26. Zhuge J. Overexpression of CYP2E1 induces HepG2 cells death by the AMP kinase activator 5'-aminoimidazole-4carboxamide-1-beta-D-ribofuranoside (AICAR). Cell Biol Toxicol. 2009; 25(3):253-263.

27. Vakana E, Altman JK, Glaser H, Donato NJ and Platanias LC. Antileukemic effects of AMPK activators on BCRABL-expressing cells. Blood. 2011; 118(24):6399-6402.

28. Robert G, Ben S, I, Puissant A, Colosetti P, Belhacene N, Gounon P, Hofman P, Bost F, Cassuto JP and Auberger P. Acadesine kills chronic myelogenous leukemia (CML) cells through PKC-dependent induction of autophagic cell death. PLoS One. 2009; 4(11):e7889.

29. Bellosillo B, Villamor N, Colomer D, Pons G, Montserrat E and Gil J. In vitro evaluation of fludarabine in combination with cyclophosphamide and/or mitoxantrone in B-cell chronic lymphocytic leukemia. Blood. 1999; 94(8):28362843.

30. Tang YC, Williams BR, Siegel JJ, Amon A: Identification of aneuploidy-selective antiproliferation compounds. Cell 2011; 144(4):499-512.

31. Ly P, Kim SB, Kaisani AA, Marian G, Wright WE and Shay JW. Aneuploid human colonic epithelial cells are sensitive to AICAR-induced growth inhibition through EGFR degradation. Oncogene. 2013; 32(26):3139-3146.

32. Roue G, Lopez-Guerra M, Milpied P, Perez-Galan P, Villamor N, Montserrat E, Campo E and Colomer D. Bendamustine is effective in p53-deficient B-cell neoplasms and requires oxidative stress and caspase-independent signaling. Clin Cancer Res. 2008; 14(21):6907-6915.

33. Theodoropoulou S, Brodowska K, Kayama M, Morizane Y, Miller JW, Gragoudas ES and Vavvas DG. Aminoimidazole carboxamide ribonucleotide (AICAR) inhibits the growth of retinoblastoma in vivo by decreasing angiogenesis and inducing apoptosis. PLoS One. 2013; 8(1):e52852.

34. Lamm W, Kaufmann H, Raderer M, Hoffmann M, Chott A, Zielinski C, Drach J. Bortezomib combined with rituximab and dexamethasone is an active regimen for patients with relapsed and chemotherapy-refractory mantle cell lymphoma. Haematologica. 2011; 96(7):1008-1014.
35. Ansell SM, Tang H, Kurtin PJ, Koenig PA, Inwards DJ, Shah K, Ziesmer SC, Feldman AL, Rao R, Gupta M, Erlichman $\mathrm{C}$ and Witzig TE. Temsirolimus and rituximab in patients with relapsed or refractory mantle cell lymphoma: a phase 2 study. Lancet Oncol. 2011; 12(4):361-368.

36. Wang $M$, Fayad L, Wagner-Bartak N, Zhang L, Hagemeister F, Neelapu SS, Samaniego F, McLaughlin P, Fanale M, Younes A, Cabanillas F, Fowler N, Newberry KJ, Sun L, Young KH, Champlin R, et al. Lenalidomide in combination with rituximab for patients with relapsed or refractory mantle-cell lymphoma: a phase $1 / 2$ clinical trial. Lancet Oncol. 2012; 13(7):716-723.

37. Cartron G, Trappe RU, Solal-Celigny P and Hallek M. Interindividual variability of response to rituximab: from biological origins to individualized therapies. Clin Cancer Res. 2011; 17(1):19-30.

38. Maloney DG. Anti-CD20 antibody therapy for B-cell lymphomas. N Engl J Med. 2012; 366(21):2008-2016.

39. Bellosillo B, Villamor N, Lopez-Guillermo A, Marce S, Esteve J, Campo E, Colomer D and Montserrat E. Complement-mediated cell death induced by rituximab in B-cell lymphoproliferative disorders is mediated in vitro by a caspase-independent mechanism involving the generation of reactive oxygen species. Blood. 2001; 98(9):2771-2777.

40. Liu J, Liu W, Ying H, Zhao W and Zhang H. Analysis of microRNA expression profile induced by AICAR in mouse hepatocytes. Gene. 2013; 512(2):364-372.

41. Walshe CA, Beers SA, French RR, Chan CH, Johnson PW, Packham GK, Glennie MJ and Cragg MS. Induction of cytosolic calcium flux by CD20 is dependent upon B Cell antigen receptor signaling. J Biol Chem. 2008; 283(25):16971-16984.

42. Franke A, Niederfellner GJ, Klein C and Burtscher H. Antibodies against $\mathrm{CD} 20$ or B-cell receptor induce similar transcription patterns in human lymphoma cell lines. PLoS One. 2011; 6(2):e16596.

43. Bonavida B. Rituximab-induced inhibition of antiapoptotic cell survival pathways: implications in chemo/ immunoresistance, rituximab unresponsiveness, prognostic and novel therapeutic interventions. Oncogene. 2007; 26(25):3629-3636.

44. Poulsen CB, Borup R, Borregaard N, Nielsen FC, Moller MB and Ralfkiaer E. Prognostic significance of metallothionein in B-cell lymphomas. Blood. 2006; 108(10):3514-3519.

45. Lewis TS, McCormick RS, Emmerton K, Lau JT, Yu SF, McEarchern JA, Grewal IS and Law CL. Distinct apoptotic signaling characteristics of the anti-CD40 monoclonal antibody dacetuzumab and rituximab produce enhanced antitumor activity in non-Hodgkin lymphoma. Clin Cancer Res. 2011; 17(14):4672-4681.

46. Chou TC and Talalay P. Quantitative analysis of dose-effect relationships: the combined effects of multiple drugs or enzyme inhibitors. Adv Enzyme Regul. 1984; 22:27-55. 
47. Saeed AI, Sharov V, White J, Li J, Liang W, Bhagabati N, Braisted J, Klapa M, Currier T, Thiagarajan M, Sturn A, Snuffin M, Rezantsev A, Popov D, Ryltsov A, Kostukovich E, et al. TM4: a free, open-source system for microarray data management and analysis. Biotechniques. 2003; 34(2):374-378.

48. Breitling R, Armengaud P, Amtmann A and Herzyk P. Rank products: a simple, yet powerful, new method to detect differentially regulated genes in replicated microarray experiments. FEBS Lett. 2004; 573(1-3):83-92.

49. Roue G, Perez-Galan P, Mozos A, Lopez-Guerra M, Xargay-Torrent S, Rosich L, Saborit-Villarroya I, Normant E, Campo E and Colomer D. The Hsp90 inhibitor IPI-504 overcomes bortezomib resistance in mantle cell lymphoma in vitro and in vivo by down-regulation of the prosurvival ER chaperone BiP/Grp78. Blood. 2011; 117(4):1270-1279. 